\title{
Ribavirin Treatment for Critically III COVID-19 Patients: An Observational Study
}

\author{
Yonghao $\mathrm{Xu}^{*}$ \\ Manshu Li $\mathbb{D}^{*}$ \\ Liang Zhou* \\ Dongdong Liu \\ Weiqun $\mathrm{He}$ \\ Weibo Liang \\ Qingwen Sun \\ Huadong Sun \\ Yimin Li \\ Xiaoqing Liu (D)
}

Department of Critical Care Medicine, State Key Laboratory of Respiratory Diseases, Guangzhou Institute of Respiratory Health, First Affiliated Hospital of Guangzhou Medical

University, Guangzhou, Guangdong,

510120, People's Republic of China

*These authors contributed equally to this work
Correspondence: Xiaoqing Liu Department of Critical Care Medicine, State Key Laboratory of Respiratory

Diseases, Guangzhou Institute of Respiratory Health, First Affiliated Hospital of Guangzhou Medical University, I5I Yanjiang Street West, Guangzhou, Guangdong, 510120, People's Republic of China

Email IxqIII8@I26.com
Background: The coronavirus disease 2019 (COVID-19) pandemic has spread all over the world resulting in high mortality, yet no specific antiviral treatment has been recommended.

Methods: A retrospective descriptive study was conducted involving 19 consecutive critically ill patients during January 27, 2020 to April 18, 2020. Ribavirin was given at $0.15 \mathrm{~g}$ q8h orally upon ICU admission for 7 to 21 days. Here, 28-day mortality, lower respiratory tract specimens (ETA), and ribavirin side effect on the day of ICU admission (Day 1), Day 7, Day 14 and Day 21 were analyzed.

Results: All the nineteen critically ill COVID-19 patients (14 males and 5 females, median age 56yr) survived through to the 28th day of observations with 6 patients $(31.58 \%)$ being discharged from the ICU. The SARS-CoV-2 viral positivity in sputum/ETA was $100 \%$ (19/ 19) on Day 1, 73.68\% (14/19) on Day 7, 57.89\% (11/19) on Day 14 and 36.84\% (7/19) on Day 21. Ribavirin side effect was not observed in these patients.

Conclusion: Ribavirin is well tolerated in critically ill patients with COVID-19 and may benefit COVID-19 patients through increasing the virus clearance.

Keywords: SARS-CoV-2, COVID-19, ribavirin, antiviral

\section{Introduction}

Severe Acute Respiratory Syndrome Coronavirus 2 (SARS-CoV-2), has resulted in a pandemic around the world known as the Coronavirus Disease 2019 (COVID-19). On July 21, 2021, more than 190 millions confirmed cases and over 4.1 millions deaths were reported. ${ }^{1}$ However, a specific antiviral agent to treat the infected is still lacking. ${ }^{2}$ Ribavirin, a broad-spectrum antiviral medication, has been utilized in clinical medicine since the early 1970s. Ribavirin exerts its anti-virus function through several mechanisms, such as inducing mutations in RNA viral genomes; by inhibiting IMP dehydrogenase or viral RNA polymerases by its metabolite RTP. ${ }^{3}$ Therefore, it has been used to treat various virus infections, such as Respiratory syncytial virus, Hepatitis $\mathrm{C}$ virus and Lassa fever infections. ${ }^{4,5}$ Additionally, its antiviral activity against severe acute respiratory syndrome (SARS) and Middle East respiratory syndrome (MERS) was also proposed and had been explored. ${ }^{6,7}$ During the emergence of SARS-CoV-2, Ribavirin was repurposed as one agent to treat COVID-19 and promising results are reported in in vitro studies. ${ }^{8,9}$

Here, we reviewed our experience of antiviral treatment using Ribavirin in treating severe and critical COVID-19 patients in our ICU. 


\section{Materials and Methods}

This retrospective descriptive study was approved by the Ethics Committee of the First Affiliated Hospital of Guangzhou Medical University (Ref. ID: [2020] No.65). This study was conducted in accordance with the Declaration of Helsinki and adhered to data confidentiality principles. The informed consent was waived as it is a retrospective and non-interventional observational study. The study included 19 consecutive critically ill patients with COVID-19 admitted to the ICU of The First Affiliated Hospital of Guangzhou Medical University during January 27, 2020, to April 18, 2020. The severity of illness is defined according to the Chinese management guideline for COVID-19 (version 8.0). ${ }^{10}$ The severe cases were defined as meeting any of the following criteria: (1) respiratory distress ( $\geq 30$ breaths/min); (2) oxygen saturation $\leq 93 \%$ at rest; (3) arterial partial pressure of oxygen $\left(\mathrm{PaO}_{2}\right) /$ fraction of inspired oxygen $\left(\mathrm{FIO}_{2}\right)$ $\leq 300 \mathrm{~mm} \mathrm{Hg}$. Critical cases were defined as meeting any of the following criteria: (1) respiratory failure and requirement of mechanical ventilation; (2) shock; (3) other organ failure that required ICU care. In our study, the criteria for ICU admission included both severe cases and critical cases.

Ribavirin was given at $0.15 \mathrm{~g} \mathrm{q} 8 \mathrm{~h}$ orally upon ICU admission for 7 to 21 days. Lower respiratory tract specimens (ETA), hemoglobin, and indirect bilirubin were tested on the day of ICU admission (Day 1), Day 7, Day 14 and Day 21. The ETA was sent to the virology laboratory of our hospital for sample processing and viral RNA extraction. Viral RNA of SARS-Cov-2 was detected according to the recommendation by the Chinese Center for Disease Control and Prevention (CDC). (https://www. chinacdc.cn/jkzt/crb/zl/szkb_11803/jszl_11815/202001/ t20200123 211378.html). The viral load was indicated as cycle threshold $(\mathrm{Ct})$ value. A positive and a negative control were included in the assay, according to the manufacturer's protocol. A Ct value of $<40$ was defined as positive for SARS-CoV-2 RNA and $>40$ was defined as negative. Samples with a $\mathrm{Ct}$ value between 37 to 40 were retested, at least twice. The statistical analyses were conducted using computerized packages (All the analyses were performed using computerized statistical packages (GraphPad, San Diego, CA). Data are presented as number (\%), and median (interquartile range) as appropriate. Data were tested for differences using a Rank sum test. For all the tests, a two-tailed $\mathrm{P}$ value $<0.05$ was considered significant.

\section{Results}

Nineteen critically ill COVID-19 patients (14 males and 5 females, median age 56yr) were enrolled during January 27, 2020, to April 18, 2020. The most common symptoms were fever (17 patients), malaise (13 patients), cough (13 patients), and dyspnea ( 9 patients). The median time from symptom onset to ICU admission was 11 days. Upon admission, APACHE II score was 17 (IQR, 9-22) and SOFA score was 7 (IQR, 3-11), while $\mathrm{PaO}_{2} / \mathrm{FiO}_{2}$ ratio was 170 (IQR, 103.5-216) mmHg. Preexisting conditions were also recorded, including hypertension (8 patients), diabetes (7 patients), coronary artery disease (4 patients), chronic pulmonary disease ( 2 patients) and chronic liver disease (2 patients). All patients have received antibiotic treatment and $68.42 \%$ (13 patients) required vasopressors treatment, while $26.32 \%$ ( 5 patients) underwent glucocorticoid therapy. Other laboratory test baselines are presented in Table 1 . With a $100 \%$ survival rate on day 28 and a discharge rate of $31.58 \%$ from the ICU. Following Ribavirin treatment, the SARS-CoV-2 viral positivity in sputum/ETA was $100 \%(19 / 19)$ on day $1,73.68 \%(14 / 19)$ on day $7,57.89 \%(11 / 19)$ on day 14 and $36.84 \%(7 / 19)$ on day 21. The Ct value is shown in Figure 1. We also evaluated the side effect of Ribavirin, hemolysis, which can be reflected by the decrease of haemoglobin levels, and the elevation of indirect bilirubin levels (Figure 1). A decreasing trend was noticed in hemoglobin levels throughout day 1 to day 21, however, no statistical significant differences was observed between day 1 and day 7. Meanwhile, indirect bilirubin levels remained unchanged throughout the study period.

\section{Discussion}

COVID-19 is spreading all over the world, yet a specific antiviral agent to optimally treat the infected, and to increase the viral clearance is still lacking. ${ }^{2}$ Especially during the first wave, little information is available for COVID-19 treatment. This retrospective study presents a cohort with 19 critically ill COVID-19 patients who were treated with oral Ribavirin during January 27 to April 18, 2020. The baseline of clinical characteristics of our study population are similar to other antiviral drug trials in ICU patients with COVID-19. ${ }^{11,12}$ However, all our patients have survived within 28 days, while mortality floats around $20 \%$ in other studies. Although multiple factors may account for this high survival rate, the effectiveness of Ribavirin should not be 
Table I Baseline Demographics of the Study Population

\begin{tabular}{|c|c|}
\hline Demographic and Comorbidities & All Patients $(n=19)$ \\
\hline Age (yr) & $56(46-68)$ \\
\hline Body mass index $\left(\mathrm{kg} / \mathrm{m}^{2}\right)$ & $24.05(22.06-27.14)$ \\
\hline \multicolumn{2}{|l|}{ Sex } \\
\hline Female, n (\%) & $5(26.32)$ \\
\hline Male, n (\%) & $14(73.68)$ \\
\hline \multicolumn{2}{|l|}{ Comorbidities } \\
\hline Hypertension, n (\%) & $8(42.11)$ \\
\hline Diabetes, n (\%) & $7(36.84)$ \\
\hline Coronary artery disease, $\mathrm{n}(\%)$ & $4(21.06)$ \\
\hline Cerebrovascular disease, $\mathrm{n}(\%)$ & $0(0.00)$ \\
\hline Chronic pulmonary disease, $\mathrm{n}(\%)$ & $2(10.53)$ \\
\hline Tuberculosis, n (\%) & $0(0.00)$ \\
\hline Chronic kidney disease, n (\%) & $0(0.00)$ \\
\hline Chronic liver disease, $n(\%)$ & $2(10.53)$ \\
\hline \multicolumn{2}{|l|}{ Onset symptoms and signs } \\
\hline Fever, n (\%) & $17(89.47)$ \\
\hline Cough, n (\%) & $13(68.42)$ \\
\hline Sputum, n (\%) & $6(31.58)$ \\
\hline Shortness of breath, $n(\%)$ & $9(47.37)$ \\
\hline Chest tightness, n (\%) & $3(15.79)$ \\
\hline Myalgia, n (\%) & $4(21.05)$ \\
\hline Malaise, n (\%) & $13(68.42)$ \\
\hline Diarrhoea, n (\%) & $0(0.00)$ \\
\hline $\begin{array}{l}\text { Duration from onset of symptoms to } \\
\text { ICU admission (days) }\end{array}$ & $11(9-13.5)$ \\
\hline SOFA Score on Day I & $7(3-11)$ \\
\hline APACHEII Score on Day I & $17(9-22)$ \\
\hline Ratio of $\mathrm{PaO}_{2}$ to $\mathrm{FiO}_{2}$, on Day I & $170(103.5-216)$ \\
\hline \multicolumn{2}{|l|}{ Laboratory tests } \\
\hline White blood cell count, $\times 10^{9}$ per $\mathrm{L}$ & $9.2(6.5-12.4)$ \\
\hline$<4$ & I (5.26\%) \\
\hline $4-10$ & II (57.90\%) \\
\hline$>10$ & 7 (36.84\%) \\
\hline Lymphocyte count, $\times 10^{9}$ per $\mathrm{L}$ & $0.4(0.2-0.5)$ \\
\hline$<1.0$ & $19(100 \%)$ \\
\hline$\geq 1.0$ & $0(0 \%)$ \\
\hline Haemoglobin, g/L & $121(1 \mid 2-134)$ \\
\hline Platelet count, $\times 10^{9}$ per $\mathrm{L}$ & $143(|| 7-2 \mid 0)$ \\
\hline$<100$ & $2(10.53 \%)$ \\
\hline$\geq 100$ & 17 (89.47\%) \\
\hline Prothrombin time, $s$ & $14.8(13.6-16.3)$ \\
\hline Activated partial thromboplastin time, $s$ & $41.4(35.8-47)$ \\
\hline D-dimer, mg/L & $1.30(0.50-4.67)$ \\
\hline Serum creatinine, $\mu \mathrm{mol} / \mathrm{L}$ & $73.1(60.9-89.7)$ \\
\hline$\leq 133$ & $3(15.79 \%)$ \\
\hline$>133$ & $16(84.21 \%)$ \\
\hline
\end{tabular}

(Continued)
Table I (Continued).

\begin{tabular}{|c|c|}
\hline Demographic and Comorbidities & All Patients (n = 19) \\
\hline Aspartate aminotransferase, U/L & $34.3(22.1-53.3)$ \\
$\leq 40$ & $12(63.16 \%)$ \\
$>40$ & $7(36.84 \%)$ \\
\hline Alanine aminotransferase, U/L & $25.5(20.1-34.1)$ \\
$\leq 50$ & $16(84.21 \%)$ \\
$>50$ & $3(15.79 \%)$ \\
\hline Total bilirubin, mmol/L & $12.7(6.9-17)$ \\
\hline Lactate dehydrogenase, U/L & $405.4(354.6-529.5)$ \\
$\leq 245$ & $0(0 \%)$ \\
$>245$ & $19(100 \%)$ \\
\hline Medications during study period-no. (\%) & $13(68.42)$ \\
Vasopressors & $19(100)$ \\
Antibiotic agent & $5(26.32)$ \\
Glucocorticoid therapy & \\
\hline Outcomes & $0(3 \%)$ \\
Mortality on day 28 & $6(38 \%)$ \\
ICU discharge within 28 days & \\
\hline
\end{tabular}

Note: All data were presented as the median (interquartile range) otherwise stated.

taken lightly. Many studies have indicated that viral load is associated with outcomes of COVID-19 patients, ${ }^{13-15}$ suggesting the significance of antiviral therapy. As a mature antiviral drug, the pharmacokinetics and bioavailability information of Ribavirin is relatively wellknown, making the application of Ribavirin more manageable.

Prior to our report, Ribavirin was employed in a Phase 2 trial in combination with Interferon beta- $1 b$ and Lopinavir-Ritonavir to treat patients with mild to moderate COVID-19. In this trial, the combination treatment group was demonstrated to be superior in reducing the viral load in all clinical species compared to LopinavirRitonavir alone. ${ }^{16}$ Moreover, a hastened clinical improvement with a shorter duration of hospital stay was observed in the triple combination group as well. ${ }^{16}$ Furthermore, in a retrospective study on Ribavirin therapy for severe COVID-19 patients, despite the use of Ribavirin is not associated with reduced mortality compared to control group, a potential signal was sent. The mortality of ribavirin group $(17.1 \%)$ was numerically lower than it in the control group (24.6\%). ${ }^{17}$ Considering that only 44 patients 

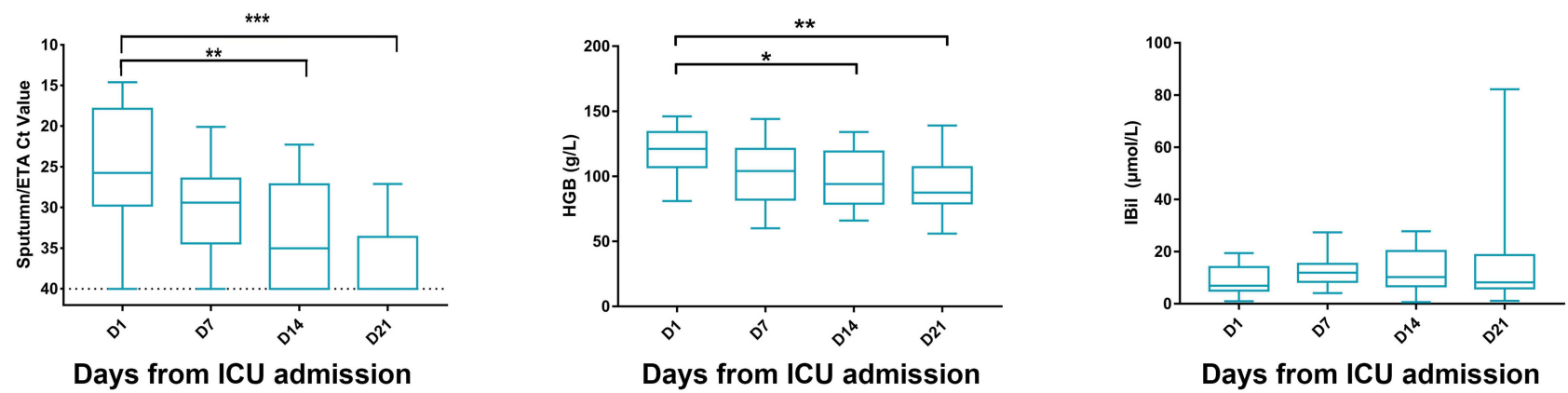

Figure I Dynamics of SARS-CoV-2 virus load and indices for hemolytic anemia during ribavirin treatment. The dynamic change of SARS-CoV-2 virus PCR's Ct values of 19 patients from sputum/ETA. HGB (hemoglobin), and IBil (indirect bilirubin) for Ribavirin-related hemolytic anemia were shown in four different time points (day I, day 7, day I4 and day 21 from ICU admission/starting Ribavirin treatment). ${ }^{*} p<0.05{ }^{* *} p<0.0$ I ${ }^{* * *} p<0.00$ I.

were treated with Ribavirin and 71 patients were served as control in this study, a larger size cohort may have enough power to detect the difference between them. In a recent published retrospective cohort study by Peng and colleagues, 403 moderate COVID-19 patients were treated with either arbidol, interferon alpha-1b, lopinavir-ritonavir or ribavirin or as combinations. ${ }^{18}$ Although Ribavirin treatment was not significantly associated with reduced risk, the trend was still positive, and it showed benefits in alleviating developing severity.

In our study, all the severe and critical COVID-19 patients received Ribavirin and reached $100 \%$ survival. In addition, the viral load of SARS-CoV-2 in lower respiratory tract and throat swabs were declining with the use of Ribavirin. On day 14, 42.11\% (8/19) and 89.47\% (17/19) patients had presented negative viral shedding in lower respiratory tract and in throat swabs respectively. The percentage of detectable SARS-CoV-2 RNA in throat swab samples of Lopinavir-Ritonavir treated, and standard care group were $55.2 \%$ and $57.1 \%$ respectively on day 14 in severe COVID-19 patients, which is much higher than it is in our study. ${ }^{11}$ Taken together, Ribavirin may play a role in reducing viral load in patients with COVID-19, therefore, resulting in a higher survival rate.

Whilst Ribavirin is showing promising results in treating COVID-19 patients, concerns on its side effect may be raised. Hemolytic anaemia is the main side effect of Ribavirin but was not common in our cohort. This is probably due to the safer dosage we chose. Although a decline of hemoglobin was observed, indirect bilirubin, another index of hemolysis had not increased. In addition, other factors may contribute to the drop of hemoglobin instead of hemolysis. 63.16\% (12/19) patients experienced gastrointestinal bleeding, $26.32 \% \quad(5 / 19)$ underwent
ECMO and $10.52 \%(2 / 19)$ were placed on CRRT. The overall results are similar to those reported by Tong et al, ${ }^{17}$ Ribavirin was well tolerated and did not lead to severe adverse events in our cohort.

This study is limited by a small sample size and no placebo controls. Furthermore, similar to most retrospective studies, with potential data sets uncollected, such as free hemoglobin was not reported. Overall, our study provides insight regarding the use and effectiveness Ribavirin brings in treating COVID-19 patients. As the world combats rippling waves of COVID-19, an accessible antiviral drug is required and further clinical trials on Ribavirin are worthy to be considered.

\section{Conclusion}

Ribavirin is well tolerated in critically ill patients with COVID-19 and may benefit COVID-19 patients through increasing the virus clearance.

\section{Abbreviations}

COVID-19, coronavirus disease 2019; ICU, intensive care unit; IQR, interquartile range; SARS-CoV-1, severe acute respiratory syndrome coronavirus 1; MERS-CoV, Middle East respiratory syndrome coronavirus; SARS-CoV-2, severe acute respiratory syndrome coronavirus 2; ETA, endotracheal aspirate; HGB, heamoglobin; IBiL, indirect bilirubin.

\section{Funding}

The study was funded by the National Natural Science Foundation of China (82070084), National Science and Technology Major Project (No. 2017ZX10204401), and the Special Project of Guangdong Science and Technology Department (2020B111105001). 


\section{Disclosure}

The authors report no conflicts of interest in this work.

\section{References}

1. World Health Organization. WHO coronavirus (COVID-19) dashboard. Available from: https://covid19.who.int/. Accessed July $21,2021$.

2. Lamontagne F, Agoritsas T, Macdonald H, et al. A living WHO guideline on drugs for covid-19. BMJ. 2020;370:m3379. doi:10.1136/ bmj.m3379

3. Parker WB. Metabolism and antiviral activity of ribavirin. Virus Res. 2005;107(2):165-171. doi:10.1016/j.virusres.2004.11.006

4. Burrows FS, Carlos LM, Benzimra M, et al. Oral ribavirin for respiratory syncytial virus infection after lung transplantation: efficacy and cost-efficiency. J Heart Lung Transplant. 2015;34(7):958-962. doi:10.1016/j.healun.2015.01.009

5. McCormick JB, King IJ, Webb PA, et al. Lassa fever. Effective therapy with ribavirin. $N$ Engl J Med. 1986;314(1):20-26. doi:10.1056/ NEJM198601023140104

6. Chu CM, Cheng VC, Hung IF, et al. Role of lopinavir/ritonavir in the treatment of SARS: initial virological and clinical findings. Thorax. 2004;59(3):252-256. doi:10.1136/thorax.2003.012658

7. Falzarano D, de Wit E, Rasmussen AL, et al. Treatment with interferon-alpha2b and ribavirin improves outcome in MERS-CoVinfected rhesus macaques. Nat Med. 2013;19(10):1313-1317. doi: $10.1038 / \mathrm{nm} .3362$

8. Elfiky AA. Ribavirin, Remdesivir, Sofosbuvir, Galidesivir, and Tenofovir against SARSCoV-2 RNA dependent RNA polymerase (RdRp): a molecular docking study.Life Sci. 2020;253:117592. doi:10.1016/j.1fs.2020.117592

9. Wang M, Cao R, Zhang L, et al. Remdesivir and chloroquine effectively inhibit the recently emerged novel coronavirus (2019-nCoV) in vitro. Cell Res. 2020;30(3):269-271. doi:10.1038/s41422-020-0282-0
10. China NHCotPsRo. Chinese management guideline for COVID-19 (version 8.0); 2020. Available from: https://www.chinadaily.com.cn/ pdf/2020/DiagnosisandTreatmentProtocolforCOVID-19Patients (Tentative8thEdition).pdf. Accessed August 18, 2020.

11. Cao B, Wang Y, Wen D, et al. A trial of lopinavir-ritonavir in adults hospitalized with severe Covid-19. $N$ Engl J Med. 2020;382 (19):1787-1799. doi:10.1056/NEJMoa2001282

12. Wang Y, Zhang D, Du G, et al. Remdesivir in adults with severe COVID-19: a randomised, double-blind, placebo-controlled, multicentre trial. Lancet. 2020;395(10236):1569-1578. doi:10.1016/ S0140-6736(20)31022-9

13. Wang Y, Zhang L, Sang L, et al. Kinetics of viral load and antibody response in relation to COVID-19 severity. J Clin Invest. 2020;130 (10):5235-5244. doi:10.1172/JCI138759

14. Pujadas E, Chaudhry F, McBride R, et al. SARS-CoV-2 viral load predicts COVID-19 mortality. Lancet Respir Med. 2020;8(9):e70. doi:10.1016/S2213-2600(20)30354-4

15. Fajnzylber J, Regan J, Coxen K, et al. SARS-CoV-2 viral load is associated with increased disease severity and mortality. Nat Commun. 2020;11(1):5493. doi:10.1038/s41467-020-19057-5

16. Hung IF, Lung K, Tso EY, et al. Triple combination of interferon beta-1b, lopinavir-ritonavir, and ribavirin in the treatment of patients admitted to hospital with COVID-19: an open-label, randomised, phase 2 trial. Lancet. 2020;395(10238):1695-1704. doi:10.1016/ S0140-6736(20)31042-4

17. Tong S, Su Y, Yu Y, et al. Ribavirin therapy for severe COVID-19: a retrospective cohort study. Int $J$ Antimicrob Agents. 2020;56 (3):106114. doi:10.1016/j.ijantimicag.2020.106114

18. Yin P, Meng J, Chen J, et al. Antiviral drugs arbidol and interferon alpha-1b contribute to reducing the severity of COVID-19 patients: a retrospective cohort study. Virol J. 2021;18(1):142. doi:10.1186/ s12985-021-01617-w
Infection and Drug Resistance

\section{Publish your work in this journal}

Infection and Drug Resistance is an international, peer-reviewed openaccess journal that focuses on the optimal treatment of infection (bacterial, fungal and viral) and the development and institution of preventive strategies to minimize the development and spread of resistance. The journal is specifically concerned with the epidemiology of

\section{Dovepress}

antibiotic resistance and the mechanisms of resistance development and diffusion in both hospitals and the community. The manuscript management system is completely online and includes a very quick and fair peerreview system, which is all easy to use. Visit http://www.dovepress.com/ testimonials.php to read real quotes from published authors. 